\title{
Calorie menu labelling in Ireland: assessment of quality and accuracy
}

\author{
J. P. Kennelly ${ }^{1}$, D. M. Logue ${ }^{1}$, E. M. Keaveney ${ }^{1}$, D. M. O’Connor ${ }^{1}$, S. M. Ní Bhriain ${ }^{1,2}$ \\ and M. A. T. Flynn ${ }^{1,2}$ \\ ${ }^{1}$ Public Health Nutrition, Food Safety Authority of Ireland, Dublin 1, Republic of Ireland and ${ }^{2}$ Northern Ireland Centre \\ for Food and Health, University of Ulster, Coleraine BT52 ISA, UK
}

Technical guidance for food businesses outlines the four best practice principles for calorie menu labelling, including that calories are displayed (1) on all standard menu items sold; (2) clearly at the 'point of choice'; (3) per portion or per meal; and (4) in terms of average daily calorie needs ${ }^{(1)}$. This study evaluates how well food service outlets claiming to display calorie information comply with these principles and the accuracy of their calorie declarations.

Dublin-based food service outlets who claimed in a telephone survey to display calorie information ${ }^{(2)}$ were assessed against the four principles using a specifically designed assessment checklist which was pre-tested for feasibility. A scoring system was developed to assess the first two principles as the proportions of standard items (principle 1), and 'points of choice' (principle 2), calorie labelled. An evaluation was conducted on whether information was provided on calories on per portion, or per meal, basis (principle 3), and on average daily calorie needs (principle 4). Three food samples (a low, medium and high calorie option) were purchased from each outlet and calorie accuracy scores were assigned as the proportion of calories displayed relative to those independently estimated using WISP (version 3). Data was analysed in PASW (version 18) where differences in all scores developed were assessed according to food business category. Calorie accuracy scores were also examined for differences according to whether the foods were low, medium or high calorie options.

\begin{tabular}{|c|c|c|c|c|}
\hline & \multicolumn{2}{|c|}{ Score on calorie labelling principles } & \multicolumn{2}{|c|}{ Calorie accuracy score } \\
\hline & $\begin{array}{c}\text { \% Standard items*labelled } \\
\text { Median (range) }\end{array}$ & $\begin{array}{c}\% \text { Points of choice } \dagger \text { labelled } \\
\text { Median (range) }\end{array}$ & $\begin{array}{l}\text { Calorie accuracy score } \neq \\
\text { Median (range) }\end{array}$ & Number of foods assessed \\
\hline Total (19) & $68(7-100)$ & $33(0-100)$ & $100(32-335)$ & 57 \\
\hline Fast-food (8) & $81(7-100)$ & $33(2-66)$ & $93(32-124)$ & 24 \\
\hline Coffee shops (9) & $64(14-93)$ & $33(0-64)$ & $107(72-335)$ & 27 \\
\hline Restaurants and Hotels (2) & $97(93-100)$ & $74(47-100)$ & $95(49-113)$ & 6 \\
\hline
\end{tabular}

* Standard item, items on sale for at least 30 days which remain the same each time they are made.

$\dagger$ Point of choice, where the food and drink are described and the price is given.

†alories displayed as a proportion (\%) of calories estimated.

As shown in the table, the proportions of standard menu items labelled varied among businesses, however overall $68 \%$ of these items displayed calorie values; while only one third of 'points of choice' were labelled. Median calorie accuracy scores were high but calorie values displayed for some food items in fast-food outlets were underestimations, while others in coffee shops were overestimations. However, there were no statistically significant differences in scores according to business category. While all outlets assessed provided calorie information on per meal, or per portion, basis; only one third of outlets $(n 7)$, of which most $(n 6)$ were fast-food outlets, provided information on average daily calorie needs.

In conclusion, food businesses displaying calorie menu labelling in Dublin provide reasonably accurate information on most of their standard menu items. However, to ensure this information is helpful for consumers, improvements are needed in calorie menu labelling at 'the point of choice' and in displaying average daily calorie requirements.

1. Food Safety Authority of Ireland (2012) at: http://www.fsai.ie/WorkArea/DownloadAsset.aspx?id=11421.

2. Logue et al. (2013). Proc Nut Soc [submitted]. 